\title{
Porous polymers via emulsion templating: pore deformation during solidification cannot be explained by an osmotic transport!
}

\author{
Lukas Koch $^{1} \cdot$ Wiebke Drenckhan ${ }^{2} \cdot$ Cosima Stubenrauch $^{1,3}$
}

Received: 13 March 2020 / Revised: 5 May 2020 / Accepted: 18 May 2020 / Published online: 3 June 2020

(C) The Author(s) 2020

\begin{abstract}
Using microfluidics, we were able to synthesize monodisperse water-in-monomer emulsions with styrene and divinylbenzene (DVB) as monomers. When polymerizing and drying these emulsions, we found that the structure of the resulting macroporous polymer strongly depends on the type of initiator. With the oil-soluble azobisisobutyronitrile (AIBN), an open-cell structure with spherical pores was obtained. However, with the water-soluble potassium peroxydisulfate (KPS), a closed-cell structure with rhombic dodecahedron-shaped pores and thick, layered pore walls was formed. In the latter case, a yet unexplained mechanism counteracts the capillary pressure arising from surface minimization: the surface area of a rhombic dodecahedron is $\sim 10 \%$ larger than that of a sphere. In our previous work, we suggested that the underlying mechanism may be osmotic transport of DVB from the plateau borders to the films. We argued that this transport also explains the layered pore walls, i.e., the formation of two outer poly-DVB-rich layers and one inner polystyrene-rich layer. In order to prove or disprove this mechanism, we carried out additional experiments. However, none of those experiments corroborated our hypothesis of osmotic transport! This study provides clear experimental evidence that our previously suggested mechanism via which spherical droplets become polyhedral pores is incorrect. We will describe (a) the rationale behind the additional experiments, (b) our expectations, and (c) our findings. Last but not least, we will discuss all of this in the light of the proposed osmotic transport.
\end{abstract}

Keywords Macroporous polystyrene $\cdot$ Microfluidics $\cdot$ Emulsion templating $\cdot$ Osmotic transport $\cdot$ Pore deformation

\section{Introduction}

Macroporous polymers (also called polymer foams) are solid materials that are composed of a continuous polymer phase and a dispersed gas phase with pore dimensions in the submillimeter range. They are typically used for thermal/acoustic insulation or packaging but are also employed in more specialized applications such as filtering or tissue engineering

Electronic supplementary material The online version of this article (https://doi.org/10.1007/s00396-020-04678-5) contains supplementary material, which is available to authorized users.

Cosima Stubenrauch

c.stubenrauch@ipc.uni-stuttgart.de

1 Institut für Physikalische Chemie, Universität Stuttgart, Pfaffenwaldring 55, 70569 Stuttgart, Germany

2 CNRS, Institute Charles Sadron UPR22, Université de Strasbourg, F-67000 Strasbourg, France

3 Institute of Advanced Studies (USIAS), Université of Strasbourg, F-67000 Strasbourg, France thanks to their adjustable density, their mechanical and chemical stability, and their high surface area [1-4]. Their properties depend on the type of polymer, on the volume of the dispersed phase, and on the foam morphology. Please note that the foam morphology is characterized by the pore size, the pore size distribution, the pore structure, and the connectivity of the pores. If macroporous polymers are synthesized in the conventional, large-scale industrial way, i.e., via foaming of a fluid polymer melt with physical or chemical blowing agents, polydisperse materials are obtained [1,2].

An increasing number of investigations deal with monodisperse macroporous polymers, i.e., materials with equalvolume pores. These materials are interesting since it is believed that their properties can be directly linked to particular pore sizes, which means that tailor-made materials can be produced. One way to produce monodisperse macroporous polymers is the use of microfluidics which allows liquid monodisperse foam and emulsion templates to be generated and then solidified [5-10]. In the case of emulsions, the dispersed phase has to be removed after the solidification of the continuous phase. Costantini et al. [11] used an oil-in-water 
emulsion to produce monodisperse, hydrophilic scaffolds based on dextran-methacrylate monomers. A hydrophobic counterpart was synthesized by Quell et al. [12-15] who used a water-in-styrene/divinylbenzene (DVB) emulsion to produce monodisperse macroporous polystyrene (PS)/polyDVB. The mass ratio between styrene and DVB was 1:1. Since the water droplets have a higher density than the continuous styrene/DVB phase, they sediment to the bottom of the vessel where they form a hexagonally close-packed structure arranged like a face-centered cubic (FCC) crystal. Note that polydisperse macroporous PS/poly-DVB synthesized via emulsion templating has been known for many years [16-21].

The morphology of the monodisperse macroporous PS/ poly-DVB reported by Quell et al. [12-15] mainly depends on the type of initiator used. It was found that the pores, just like the initial water droplets, remain spherical, in the case of the oil-soluble initiator azobisisobutyronitrile (AIBN), while they become rhombic dodecahedron-shaped (hexagonal pore cross-sections in the hexagonal plane) in the case of the watersoluble initiator potassium peroxydisulfate (KPS). Furthermore, with AIBN the spherical pores are interconnected (see Fig. 1, upper row).

The interconnections can be explained by the formation of small, flat films with thicknesses of less than $100 \mathrm{~nm}$ in the liquid state due to the drop deformation under gravity. These films are further fragilized by the shrinkage of the continuous phase during the polymerization. This shrinkage ruptures the continuous phase at its thinnest points [19]. With KPS, however, hexagonal pore cross-sections without interconnections and micrometer-thick, three-layered pore walls are created (see Fig. 1, lower row). The formation of a rhombic dodecahedron-shaped pore requires a mechanism that works against surface minimization and the resulting capillary pressure. The reason for this is that the resulting surface is $\sim 10 \%$ larger than that of the templating droplets requiring the redistribution of monomer towards the thin films [14]. With both initiators, the FCC structure remains unchanged after the emulsion template is transformed into a macroporous polymer, but the shape of the individual pore changes. To synthesize macroporous polymers with a tailored morphology, the mechanism which is responsible for the initiator-dependent morphology needs to be understood. When KPS is used as initiator, Quell et al. [13,14] suggested a mechanism that relies on two assumptions: Firstly, since the polymerization starts from the interface, a "skin" around the droplets is formed at the beginning of the polymerization. Secondly, since the cross-linker DVB is built faster into the growing polymer chain [22], the skin is primarily composed of polyDVB. The enrichment of DVB within the skin creates a concentration difference between the films (two droplets meet) where DVB is already depleted and the plateau borders (three droplets meet) where many DVB monomers are not yet polymerized. This concentration difference induces osmotic transport of DVB from the plateau borders to the films, which decreases the volume of the plateau borders and increases the volume of the films, thus leading to hexagonal pore cross-sections. The inner layer of the pore wall is formed by polymerizing the remaining styrene.

If this kind of osmotic transport were the reason for the deformation of the pores, the following trends should be observed. A systematic increase of the mass fraction of styrene would lead (1a) to a lower extent of osmotic transport and thus to increasingly spherical pores and (1b) to a decrease of the thickness of the outer layers (supposed to be mainly polyDVB) and, accordingly, to an increase of the thickness of the inner layer (supposed to be mainly PS). A systematic increase of the mass fraction of the water-soluble initiator KPS would cause (2a) the pores to become more spherical since the
Fig. 1 A monodisperse water-instyrene/DVB emulsion template (left) is polymerized leading to monodisperse macroporous PS/ poly-DVB with spherical, interconnected pore cross-sections and porous pore walls if the oilsoluble initiator AIBN is used (upper row) or hexagonal, closed pore cross-sections and layered pore walls if the water-soluble initiator KPS is used (lower row) Note that the pore cross-sections in this figure as well as in all upcoming figures are depressions

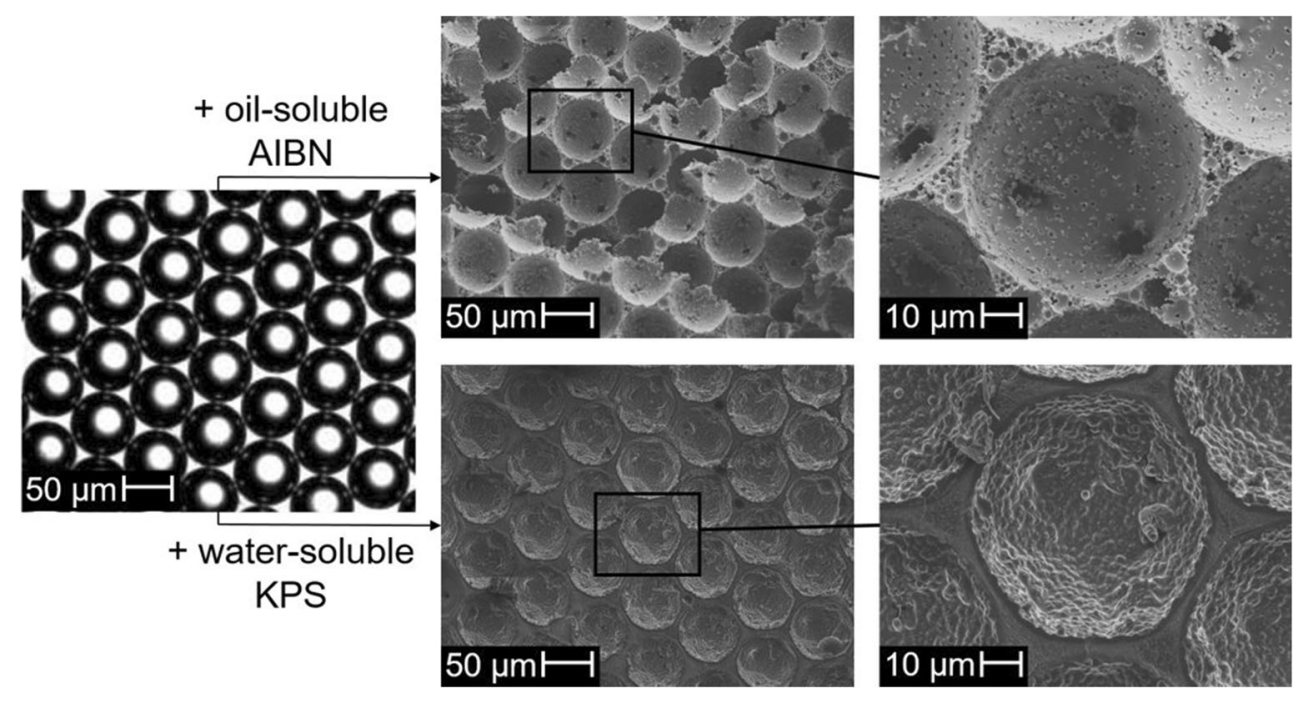


polymerization speed increases and, as a result, osmotic transport (diffusion) of DVB has less time to occur and (2b) a decrease of the visual difference between the darker outer layers and the lighter inner layer because the extent of the separation of styrene and DVB by osmotic transport decreases.

In this work, we investigate whether these trends are indeed observed experimentally. For this purpose, a first set of monodisperse macroporous PS/poly-DVB samples with styrene mass fractions of 50,60,70,80, and $90 \mathrm{wt} . \%$ (referring to the total mass of styrene and DVB) and a constant KPS mass fraction of 2.98 wt.\% (referring to the mass of $\mathrm{H}_{2} \mathrm{O}$ ) was prepared. In a second set of samples, the KPS mass fraction was varied between $0.37,0.75,1.49,2.98$, and $4.96 \mathrm{wt} . \%$ while the styrene mass fraction was kept constant at $50 \mathrm{wt} \%$. For their initial composition, Quell et al. [12-15] used a styrene mass fraction of $50 \mathrm{wt} . \%$ and a KPS mass fraction of $2.98 \mathrm{wt} . \%$. For all samples in this paper, their counterparts, i.e., monodisperse macroporous PS/poly-DVB polymerized with the oil-soluble initiator AIBN, were also synthesized. The experimental protocol we follow is exactly the one used by Quell et al. [13, 14]. We show that the mechanism proposed by Quell et al. $[13,14]$ is not in line with the results of the experiments. In other words, we prove that osmotic transport is not responsible for the structural transformation of spherical droplets into rhombic dodecahedronshaped pores.

\section{Materials and methods}

\section{Materials}

Styrene (99\%), divinylbenzene (DVB, technical grade 80\%, remaining $20 \%$ is mainly ethylstyrene), potassium peroxydisulfate (KPS, 99\%), and azobisisobutyronitrile (AIBN, 99\%) were purchased from Sigma Aldrich. The surfactant Hypermer 2296 was kindly supplied by Croda. Double-distilled water was used and all chemicals were employed without further purification.

\section{Sample preparation}

The samples were prepared in the same manner as the samples in Quell et al. [12-15]. The continuous and the dispersed phase were prepared by weighing all required components and blending them together by shaking the container until the resulting liquids were homogeneous. The emulsion templates were generated by pushing both liquids through a microfluidic chip $[12,13]$ with flow focusing geometry, hydrophobic coating, and a constriction of $100 \mu \mathrm{m} \times 105 \mu \mathrm{m}$ bought from Dolomite. The pressure controllers $O B$ I MkII (maximum pressure 2 bar) from Elveflow was used to control the pressures (and thereby the flow rates) of the two phases. The generation of the emulsion droplets and their diameter were monitored with a Nikon SMZ-800 N optical microscope coupled to an Optronis CL600X2 high speed camera. After the droplets were generated, they sedimented in a round-bottom flask to form a high internal phase emulsion (HIPE). The excess continuous phase was removed and the remaining HIPE was polymerized for $48 \mathrm{~h}$ at $70{ }^{\circ} \mathrm{C}$ in an oil bath. Finally, the samples were purified by Soxhlet extraction with ethanol for $12 \mathrm{~h}$ and dried at $60^{\circ} \mathrm{C}$ in a drying oven for $72 \mathrm{~h}$.

\section{Sample analysis}

The samples were cooled with liquid nitrogen to facilitate cutting them with a scalpel for scanning electron microscopy (SEM) analysis. A piece of roughly $5 \times 5 \times 2 \mathrm{~mm}$ was glued on an Aluminium Specimen Stub from Agar Scientific and sputtered with gold. All samples were cut and analyzed in a way that the sample surface runs through a hexagonally closepacked layer. Next, the piece was put under vacuum and microscopic pictures were taken at different magnifications with a CamScan CS 44 microscope coupled to the SEM. From the SEM pictures, the mean circumference $\langle C\rangle$ of the pore crosssections, the mean area $\langle A\rangle$ of the pore cross-sections, the mean thickness of the pore walls $d_{\text {pore wall }}$, the mean thickness of the outer layers $d_{\text {outer layer }}$ in the pore walls, and the mean thickness of the inner layer $d_{\text {inner layer }}$ in the pore walls were extracted. The image processing program ImageJ was used to obtain these values. $\langle C\rangle$ and $\langle A\rangle$ were obtained by encircling at least 50 pores at a 100 -fold magnification to enable ImageJ to calculate these values. $d_{\text {pore wall }}, d_{\text {outer layer }}$, and $d_{\text {inner layer }}$ were

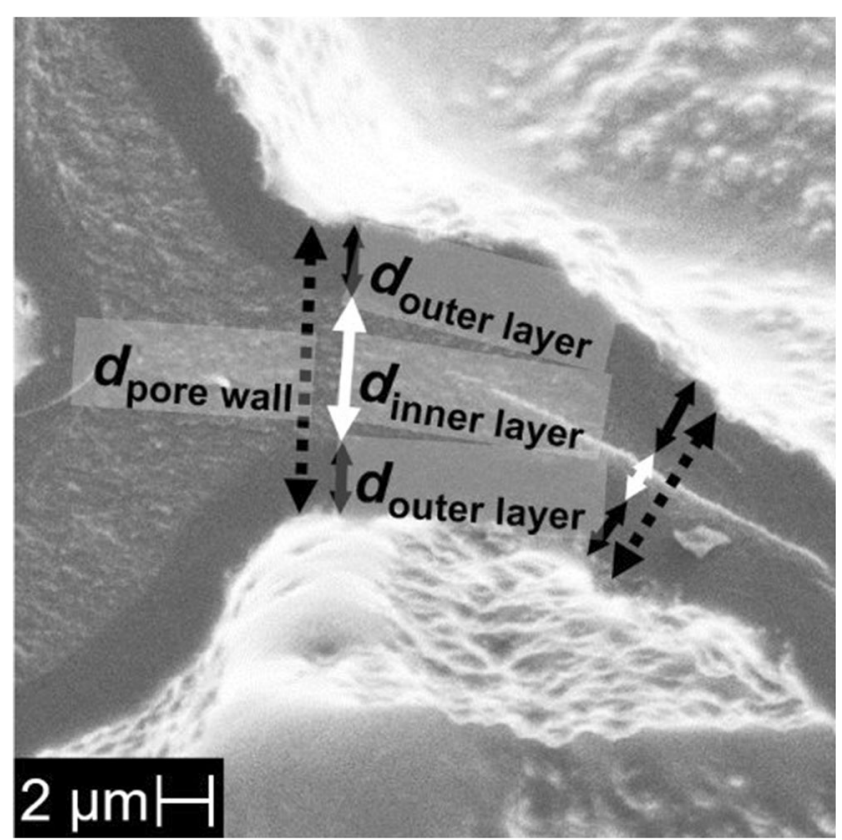

Fig. 2 Schematic representation of how $d_{\text {pore wall }}, d_{\text {outer layer, }}$ and $d_{\text {inner }}$ layer were determined 
obtained by measuring the thickness of the pore wall, of the outer layer, and of the inner layer at one common position perpendicular to the pore wall at an average of 63 locations at a 500-fold magnification. A schematic representation of how $d_{\text {pore wall }}, d_{\text {outer layer, }}$, and $d_{\text {inner layer }}$ were determined is given in Fig. 2. Figure SI-1 shows how the circumference $C$ of each pore cross-section, the area $A$ of each pore cross-section, the thickness of each pore wall $d_{\text {pore wall }}$, the thickness of each outer layer $d_{\text {outer layer in the pore wall, and the thickness of }}$ each inner layer $d_{\text {inner layer }}$ in the pore wall were determined for two exemplary SEM pictures.

\section{Results and discussion}

\section{Section 1: Variation of styrene mass fraction}

In the first set of experiments, the styrene mass fraction was varied, while the KPS mass fraction was kept constant. The styrene mass fractions (in relation to the total mass of styrene and DVB) were 50, 60, 70, 80, and $90 \mathrm{wt} . \%$. The KPS mass fraction (in relation to the total mass of $\mathrm{H}_{2} \mathrm{O}$ ) was $2.98 \mathrm{wt}$. \%. As mentioned before, the sample with a styrene mass fraction of $50 \mathrm{wt} . \%$ and a KPS mass fraction of $2.98 \mathrm{wt} . \%$ was the original composition investigated by Quell et al. [12-15]. Note that they used mol\% instead of wt.\% and thus, in their paper the molar fraction of KPS is $1.28 \mathrm{~mol} \%$. Further note that the diameter of the droplets of the water-in-styrene/DVB emulsion was always adjusted to be $72 \mu \mathrm{m}$ independent on the type and mass fraction of initiator and independent on the styrene mass fraction. A sample with pure styrene was impossible to produce since the monodisperse water-in-styrene HIPE quickly disintegrated during the heating process to $70{ }^{\circ} \mathrm{C}$ for polymerization. Note that samples containing between $0 \mathrm{wt} . \%$ styrene (pure DVB) and $40 \mathrm{wt} . \%$ styrene have morphologies similar to the sample with $50 \mathrm{wt} . \%$ styrene and will not be discussed here. Figure 3 shows the SEM pictures that were obtained for the macroporous polymers with different styrene mass fractions. All samples were cut along the main plane of the hexagonally close-packed structure ((111) plane of the FCC crystal), the cuts running through the centers of neighboring pores within the plane.

While the pictures on the left in Fig. 3 focus on the shape of the pores, the ones in the middle and especially on the right show magnifications which put in evidence the layering of the pore walls. All samples show pores which are clearly deformed with respect to the spherical pore shape of the liquid template. The pore cross-sections of the three samples with 50,60 , and $70 \mathrm{wt} . \%$ styrene all have a more or less hexagonal shape. For $50 \mathrm{wt} . \%$ styrene, this was already reported by Quell et al. [12-15]. When increasing the styrene mass fraction from 50 to $90 \mathrm{wt} . \%$, the pore cross-sections start to lose their hexagonal shape and develop into very distorted shapes. This is especially the case when the styrene mass fraction is increased from 70 to $80 \mathrm{wt} . \%$. The cross-sections of the samples with 80 and $90 \mathrm{wt} . \%$ styrene do not resemble any simple geometrical shape and their surface is even larger than that of the hexagonal cross-sections. In order to quantify how strongly the pores are deformed away from the original spherical pore, we define a dimensionless "shape-factor $S$ "

$S=\frac{1}{2 \cdot \sqrt{\pi}} \cdot \frac{\langle C\rangle}{\sqrt{\langle A\rangle}}$

where $\langle C\rangle$ is the average circumference of the pore crosssections in the (111)-plane and $\langle A\rangle$ their average area. $\langle C\rangle$ and $\langle A\rangle$ were determined in the manner described in the Materials and methods section. The pre-factor is chosen such that $S=1$ if the pore cross-section is circular (see Fig. 4, top left). If the circumference increases in relation to the area, i.e., if the shape becomes less circular, $S$ increases. For example, $S=1.05$ if the pore cross-section is a perfect hexagon (see Fig. 4, middle left). Thanks to the normalization, $S$ is independent of the actual pore size and can be used to compare different samples. The idea for a shape-factor stems from the science of foams where a similar factor is used for three-dimensional pores [23-27]. Here, we only analyze the pore cross-sections, but this analysis can be taken as representative for the 3D pore shape. The development of $S$ as function of the styrene mass fraction together with the values of $S$ for three exemplary shapes can be seen in Fig. 4.

As could already be seen visually in Fig. 3, all pore shapes are distorted away from a circular shape as $S>1$ for all samples. For 50 and $60 \mathrm{wt} . \%$, the shape of the pores is close to hexagonal. However, $S$ increases significantly when the styrene mass fraction is increased. Thus, the observations of the shape changes are in contrast to what is supposed to happen if osmotic transport was the reason for the structural transformation. We recall that an increase of the styrene mass fraction was supposed to lead to more spherical pores if osmotic transport took place. Thus, if osmotic transport were the reason for the structural transformation, $S$ should decrease towards 1.00 with increasing styrene mass fraction. This is clearly not the case! Note that $S=1.05$ for an ideal hexagon, while $S$ is roughly 1.10 for the samples with styrene mass fractions of 50 and $60 \mathrm{wt} . \%$. This can be explained by the fact that the circumference $C$ of the pores is not made up of six perfect straight edges as can be seen in Fig. 3. In fact, the edges are somewhat "crumpled", which increases the overall circumference $C$ of a pore, while its area $A$ stays roughly the same. When the oilsoluble initiator AIBN is used instead of KPS, the pores are spherical independently of the styrene mass fraction (see selected samples in Supporting Information, Fig. SI-2). The results presented above already give a strong indication that the mechanism responsible for the structural deformation is not an osmotic transport. In the following, we took a closer look at 
Fig. 3 SEM pictures of PS/polyDVB foams obtained via polymerization of monodisperse water-in-styrene/DVB emulsions with a KPS mass fraction of 2.98 wt. $\%$ and styrene mass fractions of $50 \mathrm{wt} . \%, 60 \mathrm{wt} . \%$, 70 wt. $\%, 80$ wt. $\%$, and 90 wt. $\%$

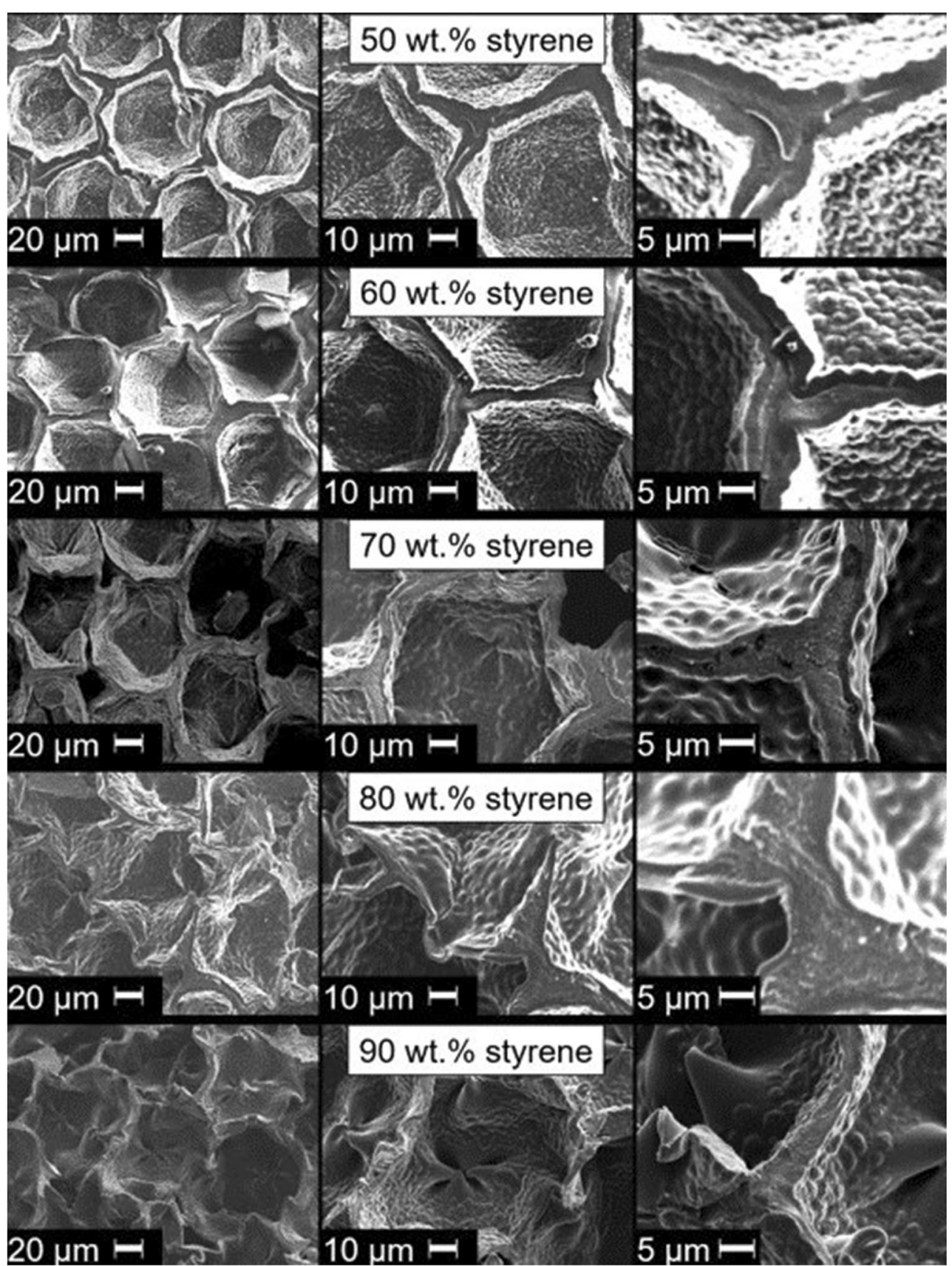

the thicknesses of the outer and inner layers as well as the ratio between them. Based on the idea of an osmotic transport, it was thought that the thickness of the outer layers (supposed to be mainly poly-DVB) should decrease and the thickness of the inner layer (supposed to be mainly PS) should increase when the styrene mass fraction is increased. The micrographs in Fig. 3 (middle) and (right) as well as the graph in Fig. 5 (top) show how the thicknesses of the pore walls and their layers depend on the styrene mass fraction. Their thicknesses were determined in the manner described in the Materials and methods section.
Looking at Fig. 5 (top), one can see that the thickness of the whole pore wall, of the outer layer, and of the inner layer all decrease when the styrene mass fraction is increased. We attribute this to the fact that the circumference of the pore crosssections increases at the same time and thus the pore walls are thinned out. We will discuss this in more detail later in connection with Fig. 5 (bottom). From Fig. 5 (top), it can be estimated that the ratio of the outer layer to the whole pore wall and the ratio of the inner layer to the whole pore wall roughly stays constant. It is interesting to note that the standard deviations of the thicknesses of the outer layers are 


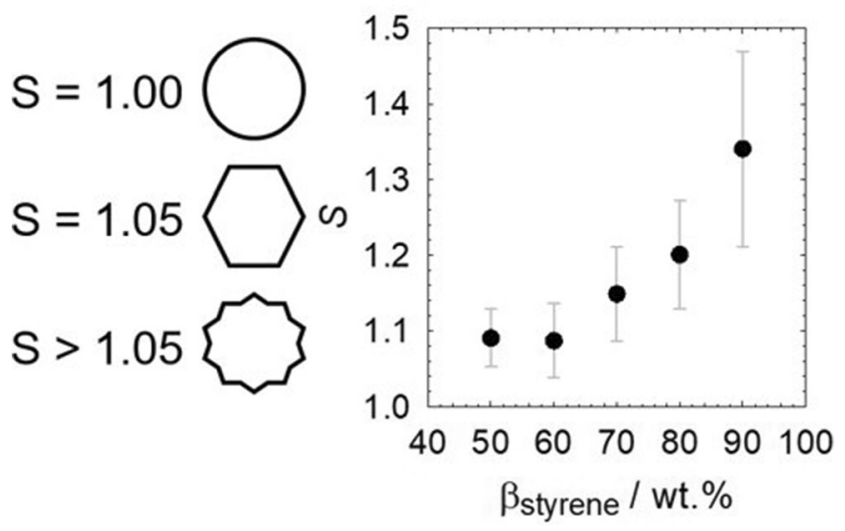

Fig. 4 (Left) Exemplary values of the shape factor $S$ for different shapes and (right) shape factor $S$ as function of the styrene mass fraction for a KPS mass fraction of $2.98 \mathrm{wt} . \%$.

considerably lower than that of the inner layers and of the whole pore wall. In other words, the values of $d_{\text {pore wall }}$ and

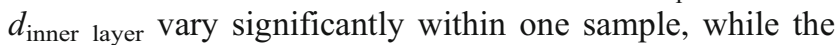
values of $d_{\text {outer layer }}$ remain roughly the same. The variation of $d_{\text {inner layer }}$ is visible in Fig. 3 (right) where the inner layer changes its thickness along the pore wall: while the inner layer is relatively thick close to the struts (former plateau borders), it becomes very thin or even vanishes completely in the pore walls. Therefore, the deviation of the mean thickness of the inner layer is higher than that of the outer layer which always has more or less the same value independent of its location. The ratio between the thickness of the outer layer to the inner layer is depicted in Fig. 5 (middle). Within the margin of error, this ratio stays constant which is a further argument against an osmotic transport being the reason for the structural deformation. We recall that the thickness of the outer layers (supposed to be mainly poly-DVB) was expected to decrease, while the thickness of the inner layer (supposed to be mainly PS) was expected to increase when the styrene mass fraction increases. Fig. 5 (middle) shows that this is clearly not the case!

As already mentioned, we attribute the overall decrease of the thickness of the whole pore wall, of the outer layer, and of the inner layer to the increase of the circumference of the pores when the styrene mass fraction is increased. In Fig. 5 (bottom), we multiplied the normalized thicknesses $d / d_{0}$ of the whole pore wall, of the outer layer, and of the inner layer by the squared normalized shape factor $S / S_{0}$ and plotted this value vs. the styrene mass fraction. The normalizing values were the thicknesses $d_{0}$ and the shape factor $S_{0}$, respectively, at a styrene mass fraction of $50 \mathrm{wt} . \%$. The thicknesses $d$ and the shape factor $S$ were normalized to end up at one common value for the whole pore wall, the outer layer, and the inner layer, respectively. The results shown in Fig. 5 (bottom) indicate that the total volume of the pore wall, of the outer layer, and of the inner layer stay roughly the same with increasing styrene mass fraction. The normalized shape
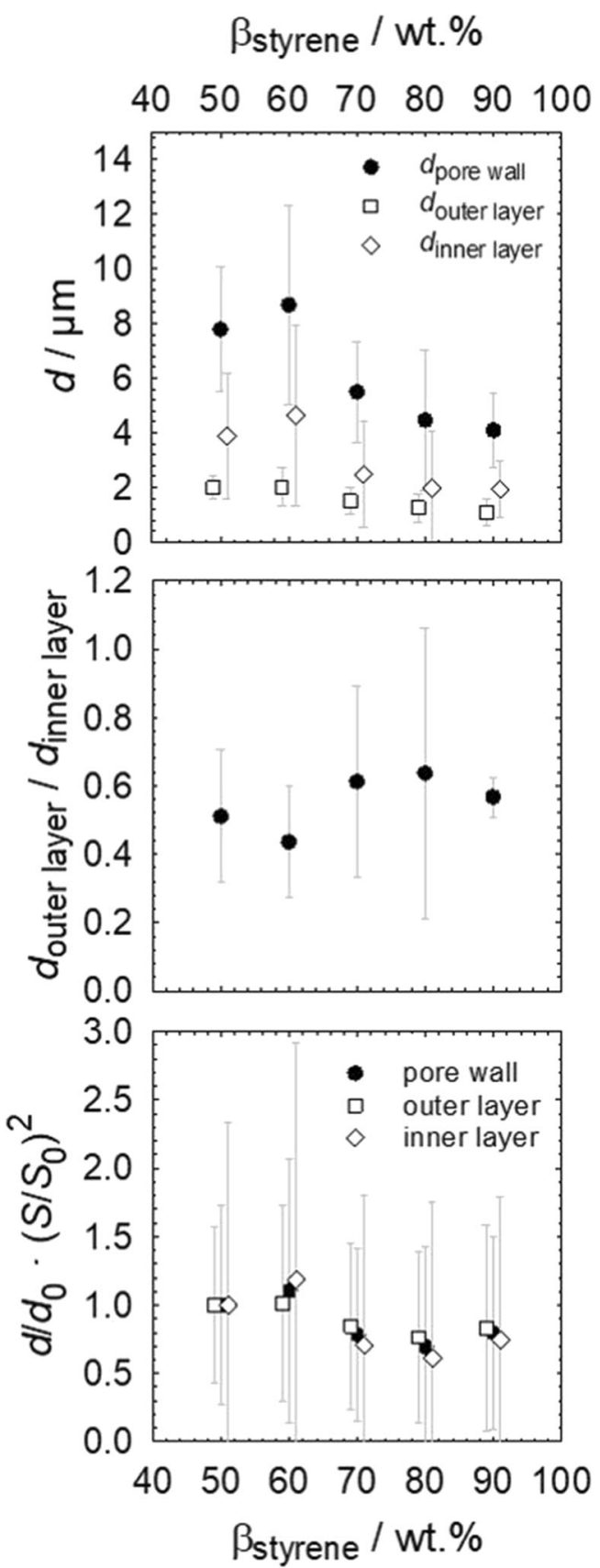

Fig. 5 (Top) Pore wall thicknesses $d_{\text {pore wall, }}$ outer layer thicknesses $d_{\text {outer }}$ layer, and inner layer thicknesses $d_{\text {inner layer }}$ as function of the styrene mass fraction for a KPS mass fraction of $2.98 \mathrm{wt}$.\%. (Middle) Ratio of $d_{\text {outer layer }}$ to $d_{\text {inner layer }}$ as function of the styrene mass fraction for a KPS mass fraction of 2.98 wt.\%. (Bottom) Normalized thicknesses $d / d_{0}$ of the whole pore wall, of the outer layer, and of the inner layer multiplied by the squared normalized shape factor $S / S_{0}$ as function of the styrene mass fraction for a KPS mass fraction of $2.98 \mathrm{wt}$. $\%$. The normalizing values were the thicknesses $d_{0}$ and the shape factor $S_{0}$, respectively, at a styrene mass fraction of $50 \mathrm{wt} . \%$. Note that in the top and bottom graphs, the data for $d_{\text {outer layer and }} d_{\text {inner layer }}$ are slightly shifted to the left and right, respectively, for the sake of clarity.

factor $S / S_{0}$ is squared because the volumes of the pore wall, of the outer layer, and of the inner layer are the most crucial values. The volume is given by the thickness of 
Fig. 6 SEM pictures of PS/polyDVB foams obtained via polymerization of monodisperse water-in-styrene/DVB emulsions with a styrene mass fraction of $50 \mathrm{wt} . \%$ and KPS mass fractions of 0.37 wt. $\%, 0.75$ wt. $\%$, 1.49 wt. $\%, 2.98$ wt. $\%$, and 4.96 wt. $\%$
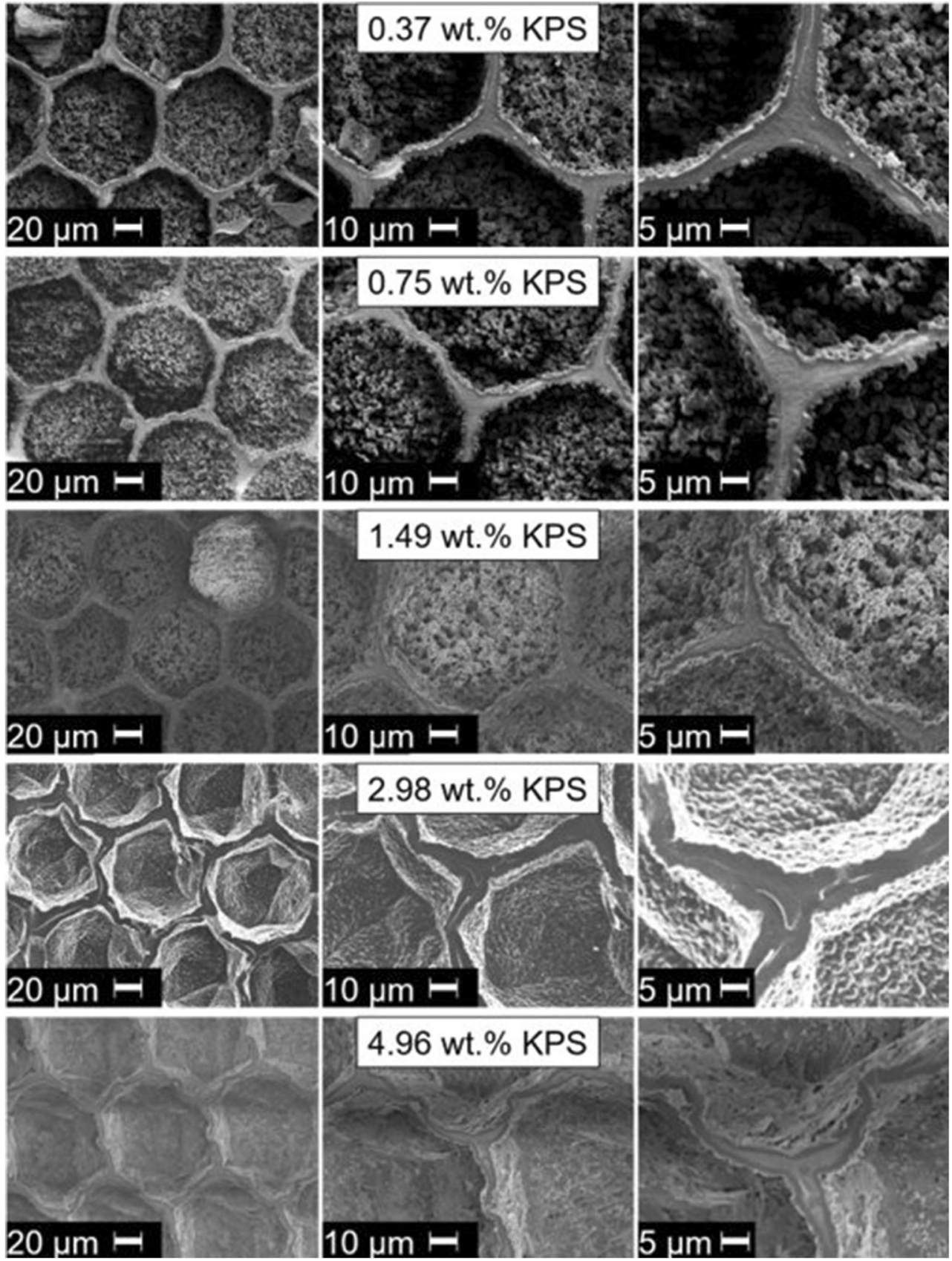

the wall or layer, respectively, multiplied by the surface area of the pore. As the shape factor $S$ is measured in 2D, it has to be squared to resemble the surface area. One can see that the values in Fig. 5 (bottom) remain more or less constant and close to one, thus supporting our explanation that the pore wall is thinned out when the circumference of the pore cross-sections is increased as the total amount of material is finite. The large error bars are due to the fact that when calculating the values, the errors of four parameters $\left(d, d_{0}, S\right.$, and $\left.S_{0}\right)$ have to be taken into account which drastically increases the overall error. From these measurements we can conclude that the mechanism which creates the visible layers seems to be identical for all formulations, the only parameter which changes is the total surface area of the pores which increases with increasing styrene mass fraction.

\section{Section 2: Variation of KPS mass fraction}

In the second set of experiments, the KPS mass fraction was varied, while the styrene mass fraction was kept constant at 50 wt. \%. The KPS mass fractions were $0.37,0.75,1.49,2.98$, 


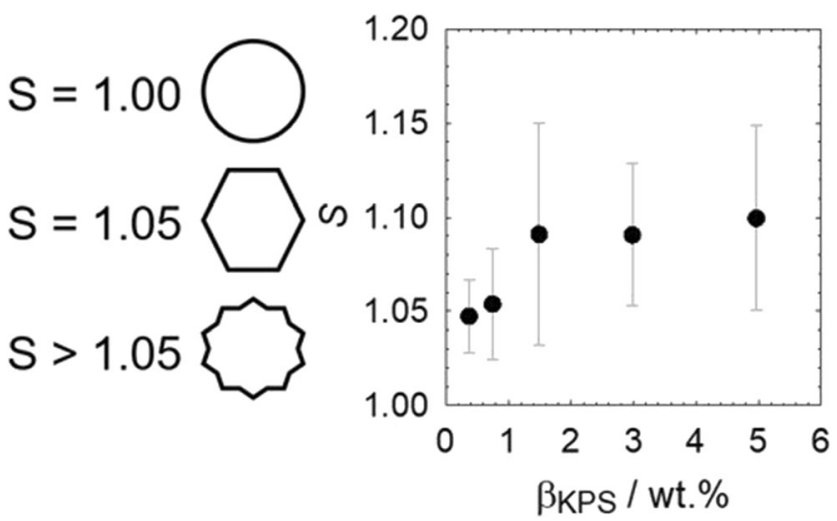

Fig. 7 (Left) Exemplary values of the shape factor $S$ for different shapes and (right) shape factor $S$ as function of the KPS mass fraction for a styrene mass fraction of $50 \mathrm{wt} . \%$.

and 4.96 wt.\%. The highest mass fraction reflects the maximum solubility of KPS in water [28]. Going back to the idea of osmotic transport, the pores should become more spherical if the KPS mass fraction is increased since osmotic transport (diffusion) would have less time to occur. On the other hand, if the KPS mass fraction is decreased from the reference formulation, we expected that the pore cross-sections remain hexagons. The SEM pictures in Fig. 6 (left) and the graph in Fig. 7 depict the development of the general pore shapes and the associated shape-factors $S$ as function of the KPS mass fraction.

These results are again not in line with the idea of osmotic transport since the pores have a hexagonal shape independently on the KPS mass fraction (Fig. 6 (left)). The shape-factor $S$ slightly increases when the KPS mass fraction is increased (Fig. 7). Again, the reason for this is the crumpled nature of the edges of the pores. For small KPS mass fractions ( $0.37 \mathrm{wt} . \%$ and $0.75 \mathrm{wt} . \%)$, the edges are nearly perfectly straight and thus the pores resemble ideal hexagons with $S$-values close to 1.05 . For higher KPS mass fractions, on the other hand, the edges are crumpled which increases $S$ to 1.10. Figure 6 (middle) and (right) further show that at small KPS mass fractions the pores are filled with many small particles, while for high KPS mass fractions the pores are empty. We attribute this observation to two competing polymerization processes. The first one is the polymerization of the continuous phase which starts at the water-styrene/DVB interface. The second one is the polymerization of monomers that are dissolved in water. Even if the solubility of styrene and DVB in water is small, it is not zero $[29,30]$ and thus, polymer particles can form in the dispersed phase via emulsion polymerization. If the KPS mass fraction is small, the polymerization of the continuous phase is slow and the polymerization of polymer particles in the dispersed phase has a longer timespan to occur. If the KPS mass fraction is high, on the other hand, the polymerization of the continuous phase is fast and hence, only a small number of polymer particles are formed (visible on pore wall surface).

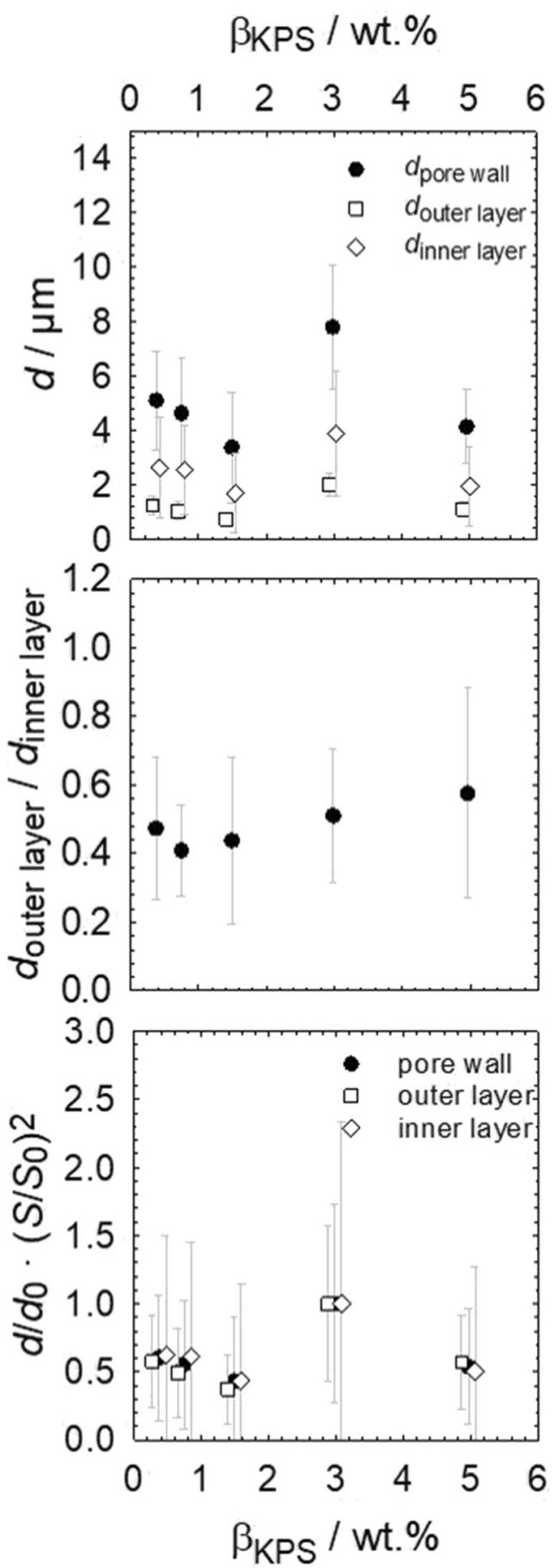

Fig. 8 (Top) Pore wall thicknesses $d_{\text {pore wall, }}$ outer layer thicknesses $d_{\text {outer }}$ layer, and inner layer thicknesses $d_{\text {inner layer }}$ as function of the KPS mass fraction for a styrene mass fraction of $50 \mathrm{wt}$.\%. (Middle) Ratio of $d_{\text {outer }}$ layer to $d_{\text {inner layer }}$ as function of the KPS mass fraction for a styrene mass fraction of $50 \mathrm{wt}$.\%. (Bottom) Normalized thicknesses $d / d_{0}$ of the whole pore wall, of the outer layer, and of the inner layer multiplied by the squared normalized shape factor $S / S_{0}$ as function of the KPS mass fraction for a styrene mass fraction of $50 \mathrm{wt}$.\%. The normalizing values were the thicknesses $d_{0}$ and the shape factor $S_{0}$, respectively, at a KPS mass fraction of $2.98 \mathrm{wt} . \%$. Note that in the top and bottom graphs, the data for $d_{\text {outer layer }}$ and $d_{\text {inner layer are slightly shifted to the left and right, respec- }}$ tively, for the sake of clarity 
Note that the solubility of ammonium peroxydisulfate (APS) in water [31] is a factor of 12.4 times higher than that of KPS. We thus used APS to synthesize monodisperse water-in-styrene/ DVB emulsions where the APS mass fraction was varied between a minimum of $0.32 \mathrm{wt} . \%$ and a maximum of 39.44 wt.\% (see Fig. SI-3 in Supporting Information). We conducted this set of experiments to test whether the pore crosssections kept their hexagonal shape when the initiator mass fraction was increased to much higher values than the reference formulation with a similar initiator. Even when the APS mass fraction was raised up to $19.72 \mathrm{wt} \%$, the pore cross-sections were still hexagonal. However, the pores became increasingly polydisperse when the APS mass fraction was raised to high levels. At the highest APS mass fraction of $39.44 \mathrm{wt} . \%$, the pores became interconnected to our surprise. Note that we assumed that the difference in reactivity between KPS and APS is negligible small, but did not conduct experiments on this matter. When the oil-soluble initiator AIBN is used instead of KPS, the pores are spherical independent on the AIBN mass fraction (see selected samples in Fig. SI-2 in Supporting Information).

From the SEM pictures in Fig. 6 (middle) and (right) the graph in Fig. 8 (top), conclusions on the development of the thickness of the pore walls, of the outer layers, and of the inner layers can be drawn. As was the case in section 1, all thicknesses (see Fig. 8 (top)) and especially the ratio between the thickness of the outer layer to that of the inner layer (see Fig. 8 (middle)) stay roughly the same. In addition, the thickness of the outer layer again has a small standard deviation for each sample, while the thicknesses of the whole pore wall and of the inner layer have a high standard deviation. In other words, the determined thicknesses and their ratios are approximately the same for all shown samples, i.e., they are roughly independent on the KPS mass fraction. Looking at Fig. 8 (bottom), one can see that $d / d_{0}$ multiplied by $S / S_{0}$ again results in constant values that are around 0.5 . The exception to this are the values at a KPS mass fraction of $2.98 \mathrm{wt} . \%$ which are 1 since the normalizing thicknesses $d_{0}$ and the normalizing shape factor $S_{0}$ also stem from this KPS mass fraction. Since the whole pore wall, the outer layer, and the inner layer were all considerably thicker at a KPS mass fraction of $2.98 \mathrm{wt} . \%$ than at the other four mass fractions (see Fig. 8 (top)), the product of $d / d_{0}$ multiplied by $S / S_{0}$ is smaller than 1 for these samples and not close to 1 as in Fig. 5 (bottom). However, since the values of $d / d_{0}$ multiplied by $S / S_{0}$ in these four samples are close to each other, this means that the total volume of the whole pore wall, of the outer layer, and the inner layer in these samples does not change significantly when the KPS mass fraction is varied.

\section{Conclusion}

In this paper we showed via two sets of experiments that osmotic transport as described by Quell et al. [14] cannot be the reason for the transformation of spherical droplets into rhombic dodecahedron-shaped pores (hexagonal pore crosssections). (1) If osmotic transport were the reason for the transformation, the pores would become more spherical with increasing styrene mass fraction. However, when the styrene mass fraction was increased from $50 \mathrm{wt} . \%$ to $90 \mathrm{wt} . \%$, the pores became even more deformed meaning that the surfaceto-volume ratio increased. Furthermore, based on the hypothesis of osmotic transport, the outer layer of the pore wall was supposed to become thinner and the inner layer thicker with increasing styrene mass fraction. However, the thickness of both the outer and the inner layers remained constant. (2) If osmotic transport were the reason for the transformation, the pores would become more spherical with increasing KPS mass fraction. However, when the KPS mass fraction was increased from 2.98 to 4.96 wt. $\%$, the pore cross-sections remained hexagonal. When the KPS mass fraction was decreased to $0.37 \mathrm{wt} . \%$, the pore cross-sections remained hexagonal as well. With low KPS mass fractions, the edges of the pores were very straight and the pores were filled with small particles. With higher KPS mass fractions, the edges of the pores become "crumpled" and the pores were empty. When the KPS mass fraction was varied, the ratio of the outer to the inner layers of the pore wall stayed constant. In all cases, the counterparts that were polymerized with the oil-soluble initiator AIBN resulted in porous polymers with spherical and interconnected pores without any layering of the pore walls. After disproving the preexisting hypothesis, our current focus lies on identifying and proving the actual mechanism that is responsible for the structural transformation.

Acknowledgments Open Access funding provided by Projekt DEAL. We would like to thank Sabrina Beyer and Benjamin Kordes for the preparation of the samples. We would like to thank the Institute for Combustion Technology from the University of Stuttgart for providing the SEM and Dr. Fels for his support of the measurements. Part of this work has been conducted within the IdEx Unistra framework (Chair W. Drenckhan) and has, as such, benefited from funding from the state, managed by the French National Research Agency as part of the "Investments for the future" program. The work was also supported by an ERC consolidator grant (agreement 819511-METAFOAM). Cosima Stubenrauch acknowledges a fellowship by the Institute of Advanced Studies at the University of Strasbourg (USIAS).

Authors' contributions Lukas Koch: experiments, analysis, and original draft. Wiebke Drenckhan: supervision and editing of the manuscript. Cosima Stubenrauch: supervision and editing of the manuscript.

\section{Compliance with ethical standards}

Conflict of interest The authors declare no conflict of interests.

Open Access This article is licensed under a Creative Commons Attribution 4.0 International License, which permits use, sharing, adaptation, distribution and reproduction in any medium or format, as long as you give appropriate credit to the original author(s) and the source, provide a link to the Creative Commons licence, and indicate if 
changes were made. The images or other third party material in this article are included in the article's Creative Commons licence, unless indicated otherwise in a credit line to the material. If material is not included in the article's Creative Commons licence and your intended use is not permitted by statutory regulation or exceeds the permitted use, you will need to obtain permission directly from the copyright holder. To view a copy of this licence, visit http://creativecommons.org/licenses/by/4.0/.

\section{References}

1. Landrock AH (1995) Handbook of plastic foams - types, properties, manufacture and applications. Noyes Publications, Park Ridge

2. Lee ST, Park CB, Ramesh NS (2007) Polymeric foams. Taylor \& Francis Group, Boca Raton

3. Mills N (2007) Polymer foams handbook: engineering and biomechanics applications and design guide. Elsevier, Amsterdam

4. Freed LE, Vunjak-Novakovic G, Biron RJ, Eagles DB, Lesnoy DC, Barlow SK, Langer R (1994) Biodegradable polymer scaffolds for tissue engineering. Nat Biotechnol 12:689-693

5. Chung KY, Mishra NC, Wang CC, Lin FH, Lin KH (2009) Fabricating scaffolds by microfluidics. Biomicrofluidics 3 : 022403-(1-8)

6. Choi SW, Cheong IW, Kim JH, Xia Y (2009) Preparation of uniform microspheres using a simple device and their crystallization into close-packed lattices. Small 5(4):454-459

7. Lin JY, Lin WJ, Hong WH, Hung WC, Nowotarski SH, Montenegro Gouveai S, Cristo I, Lin KH (2011) Morphology and organization of tissue cells in 3D microenvironment of monodisperse foam scaffolds. Soft Matter 7:10010-10016

8. Wang CC, Yang KC, Lin KH, Liu HC, Lin FH (2011) A highly organized three-dimensional alginate scaffold for cartilage tissue engineering prepared by microfluidic technology. Biomaterials 32:7118-7126

9. Sun YS, Peng SW, Lin KH, Cheng JY (2012) Electrotaxis of lung cancer cells in ordered three-dimensional scaffolds. Biomicrofluidics 6:014102-(1-14)

10. Colosi C, Costantini M, Barbetta A, Pecci R, Bedini R, Dentini M (2013) Morphological comparison of PVA scaffolds obtained by gas foaming and microfluidic foaming techniques. Langmuir 29: 82-91

11. Costantini M, Colosi C, Guzowski J, Barbetta A, Jaroszewicz J, Swieszkowski W, Dentini M, Garstecki P (2014) Highly ordered and tunable polyHIPEs by using microfluidics. J Mater Chem B 2: 2290-2300

12. Quell A, Elsing J, Drenckhan D, Stubenrauch C (2015) Monodisperse polystyrene foams via microfluidics - a novel templating route. Adv Eng Mater 17:604-609

13. Quell A, de Bergolis B, Drenckhan W, Stubenrauch C (2016) How the locus of initiation influences the morphology and the pore connectivity of a monodisperse polymer foam. Macromolecules 49:5059-5067

14. Quell A, Heitkam S, Drenckhan W, Stubenrauch C (2017) Creating honeycomb structures in porous polymers by osmotic transport. ChemPhysChem 18:451-454

15. Quell A, Sottmann T, Stubenrauch C (2017) Diving into the finestructure of macroporous polymer foams synthesized via emulsion Templating: a phase diagram study. Langmuir 33:537-542

16. Bartl H, von Bonin W (1962) Über die Polymerisation in umgekehrter Emulsion. Macromol Chem Phys 57:74-95

17. Williams JM, Wrobleski DA (1988) Spatial distribution of the phases in water-in-oil emulsions. Open and closed microcellular foams from cross-linked polystyrene. Langmuir 4:656-662

18. Hainey P, Huxham IM, Rowatt B, Sherrington DC, Tetley L (1991) Synthesis and Ultrastructural studies of styrene-divinylbenzene polyhipe polymers. Macromolecules 24:117-121

19. Cameron NR, Sherrington DC, Albiston L, Gregory DP (1996) Study of the formation of the open-cellular morphology of poly(styrene/divinylbenzene) polyHIPE materials by cryo-SEM. Colloid Polym Sci 274(6):592-595

20. Tai H, Sergienko A, Silverstein MS (2001) Organic-inorganic networks in foams from high internal phase emulsion polymerizations. Polymer 42:4473-4482

21. Menner A, Powell R, Bismarck A (2006) A new route to carbon black filled polyHIPEs. Soft Matter 2:337-342

22. Schwachula G (1975) Calculation of the copolymerization parameters in the ternary copolymerization system of styrene/m-divinylbenzene/p-divinylbenzene. J Polym Sci Polym Symp 53:107-112

23. Kraynik AM, Reinelt DA, van Swol F (2004) Structure of random foam. Phys Rev Lett 93(20):208301-(1-4)

24. Hilgenfeldt S, Kraynik AM, Reinelt DA, Sullivan JM (2004) The structure of foam cells: isotropic plateau polyhedra. EPL 67(3): 484-490

25. Kraynik AM, Reinelt DA, van Swol F (2005) Structure of random bidisperse foam. Colloids Surf A Physicochem Eng Asp 263:11-17

26. Kraynik AM (2006) The structure of random foam. Adv Eng Mater 8(9):900-906

27. Drenckhan W, Hutzler S (2015) Structure and energy of liquid foams. Adv Colloid Interf Sci 224:1-15

28. http://gestis.itrust.de/nxt/gateway.dll/gestis_de/001180.xml?f= templates $\$$ fn $=$ default.htm $\$ 3.0(04.05 .2020)$

29. http://gestis.itrust.de/nxt/gateway.dll/gestis_de/010110.xml?f= templates $\$ \mathrm{fn}=$ default.htm $\$ 3.0(04.05 .2020)$

30. http://gestis.itrust.de/nxt/gateway.dll/gestis_de/038850.xml?f= templates $\$$ fn $=$ default.htm $\$ 3.0(04.05 .2020)$

31. http://gestis.itrust.de/nxt/gateway.dll/gestis_de/002310.xml?f= templates $\$$ fn $=$ default.htm $\$ 3.0(04.05 .2020)$

Publisher's note Springer Nature remains neutral with regard to jurisdictional claims in published maps and institutional affiliations. 